gesichert und der wissenschaftliche Nachwuchs gefördert werden. Auf der einen Seite haben die Hochschulen wegen des engen personellen Spielraums kaum Möglichkeiten, aus dem eigenen Personalbestand durch Umwidmungen forschungsaktive Fächer zu stärken, Schwerpunkte aufzubauen und neue forschungsintensive Fachgebiete aufzugreifen. Auf der anderen Seite haben selbst fähigste Nachwuchswissenschaftler nur geringe Chancen, eine Professorenstelle zu erhalten; sie suchen deshalb berufliche Alternativen außerhalb der Forschung.

Die Präsidenten beziehungsweise Vorsitzenden der MaxPlanck-Gesellschaft, der Deutschen Forschungsgemeinschaft, der Westdeutschen Rektorenkonferenz, der Arbeitsgemeinschaft der Großforschungseinrichtungen, des Wissenschaftsrates und der Fraunhofer-Gesellschaft schlagen deshalb in einem gemeinsamen Memorandum vom 16. Oktober ein Programm für die Einrichtung von zentralen Pools für Professorenstellen bei den Wissenschaftsministerien der Länder vor. Zum Ausgleich der verzerrten Altersstruktur sollen bis zum Ende dieses Jahrzehnts jährlich mindestens 200 neue Professorenstellen geschaffen werden, die den Hochschulen gezielt unter forschungspolitischen Gesichtspunkten zugewiesen werden. Von Mitte der 90er Jahre an soll dann von der wieder größer werdenden Zahl der Professorenstellen jährlich ein Teil eingezogen werden. Dieser Überbrükkungsplan, dessen finanzielle Auswirkungen überschaubar seien, lehnt sich an den schon seit Jahren von der Westdeutschen Rektorenkonferenz vertretenen FiebigerPlan an.

\section{BMFT: Schwerpunkt Materialforschung}

Als ein neuer Schwerpunkt der staatlichen Forschungsförderung stellt sich immer deutlicher die Materialforschung heraus. 1985 will das Bundesforschungsministerium ein entsprechendes Programm beginnen. Unter dem Stichwort „Materialforschung - Strukturmaterialien" sollen Vorhaben auf den Gebieten Hochleistungskeramik, Pulvermetallurgie, Hochleistungspolymere, Verbundwerkstoffe und metallische Hochtemperaturwerkstoffe gefördert werden. Im Rahmen der Verbundforschung sollen dabei Institute der angewandten Grundlagenforschung mit Industrielabors zusammenarbeiten. Materialforschung außerhalb der Strukturmaterialien - Funktionswerkstoffe für die Mikroelektronik, Energieumwandlung und andere physikalische Anwen- dungen - wird verstärkt in den entsprechenden Fachprogrammen des Bundesforschungsministeriums gefördert.

\section{Trans-Schall-Windkanal und ESRF}

Die Standorte für zwei künftige europäische Großprojekte der Grundlagenforschung liegen jetzt fest. Die Bundesrepublik Deutschland und Frankreich haben sich, wie erwartet, darauf geeinigt, daß der Europäische TransSchall-Windkanal im Forschungszentrum Köln-Porz der Deutschen Forschungs- und Versuchsanstalt für Luftund Raumfahrt (DFVLR) errichtet wird und daß die Europäische Synchrotron-Strahlungsquelle (ESRF) nach Frankreich kommt. Vor allem um die ESRF hatten sich mehrere Länder und Städte in Europa beworben: Dortmund und Saarbrücken in der Bundesrepublik, Triest in Italien, unterstützt von Österreich, sowie Dänemark. In Frankreich war zunächst Straßburg der offizielle Kandidat der Regierung, doch wird die ESRF jetzt in Grenoble errichtet, wo sich das deutsch-französischbritische Institut Max von Laue-Paul Langevin (ILL) mit seinem Höchstflußreaktor beworben hatte.

Diese Entscheidungen sind nicht unumstritten. Die anderen europäischen Länder fühlen sich bei dem „,Deal““ zwischen Frankreich und Deutschland ausgetrickst, und für die deutschen Forscher, die an der ESRF arbeiten wollen, wäre Straßburg der günstiger gelegene Ort - ein Umstand, der auch unter Reisekosten-Gesichtspunkten ins Gewicht fällt.

Die Erforschung kondensierter Materie mit Hilfe der Synchrotronstrahlung gilt als ein zukunftsträchtiger interdisziplinärer Wissenschaftszweig. Sie erfaßt nicht nur Fragen der Festkörperphysik, Chemie, Kristallographie, Mineralogie, Metallkunde und Polymerforschung, sondern zunehmend auch die Molekularbiologie.

Der wissenschaftliche Bedarf an einer neuen Strahlungsquelle ist in ganz Europa in den letzten Jahren so gewachsen, daß auch der deutsche Gutachterausschuß für Großgeräte (,Pinkau-Ausschuß $\beta^{\circ}$ ) vor einem Jahr seine Zurückhaltung aufgab und darauf drängte, die ursprünglich von der Europäischen Wissenschaftsstiftung (ESF) vorgeschlagene ESRF jetzt zu beschließen. Den Forschern in der Bundesrepublik stehen als Synchrotron-Strahlungsquellen zur Zeit HASYLAB mit dem Speicherring DORIS des Deutschen Elektronen-Synchrotrons (DESY) in Hamburg, die Berliner Elektronenspeicherring-Gesellschaft (BESSY) und eine Strahlungsquelle in der Universität Bonn zur Verfügung.

Im Trans-Schall-Windkanal sollen die Flugbedingungen in der Atmosphäre an Modellen realistisch simuliert werden. 